\title{
Chronic Non-Cancer Pain: A Siren for Primary Care - A Report From the PRImary care MultiEthnic Network (PRIME Net)
}

\author{
Robert R. Leverence, MD, Robert L. Williams, MD, MPH, Michael Potter, MD, \\ Douglas Fernald, MA, Mark Unverzagt, MD, Wilson Pace, MD, Bennett Parnes, MD, \\ Elvan Daniels, MD, Betty Skipper, PhD, Robert J. Volk, PhD, Anthony E. Brown, MD, \\ and Robert L. Rhyne, MD, on behalf of PRIME Net clinicians
}

Introduction: Patients with chronic non-cancer pain (CNCP) are common and have a high degree of morbidity. Previous studies document clinician frustration and variability in CNCP management. We conducted this study to gather in-depth clinicians' views about factors that affect management of CNCP.

Methods: We conducted a survey in the Primary Care MultiEthnic Network, a consortium of PBRNs of primary care clinicians practicing in low-income, medically underserved communities, and in a network of private primary care offices.

Results: of 792 clinicians surveyed, 497 (63\%) participated. Responses and accompanying narrative comments clustered around 5 themes: (1) barriers to and uncertainties in optimal management; (2) the complex biopsychosocial nature of CNCP; (3) seriousness of prescription opioid abuse; (4) effort and burden required to properly manage $\mathrm{CNCP}$; and (5) clinician commitment to provide care for CNCP patients and benefits of expanded care model for CNCP. One-third reported a severe outcome (death or life-threatening event) in a CNCP patient for whom they had prescribed opioids. Roughly one-third do not initiate prescribing of opioids.

Conclusions: Guidelines and increased continuing medical education alone are unlikely to be the solutions to the challenges of CNCP management. Increased evidence for recommendations and resources for more comprehensive care management are needed. (J Am Board Fam Med 2011;24: 551-561.)

Keywords: Pain, Practice-based Research, Primary Health Care, Underserved Populations

More than 60 million Americans have some type of persistent or recurrent pain (chronic non-cancer pain) sufficient to substantially affect their lives. ${ }^{1,2}$ At least $40 \%$ of patients with chronic pain treated

This article was externally peer reviewed.

Submitted 29 January 2011; revised 16 May 2011; accepted 23 May 2011.

From PRIME Net Consortium; the Department of Medicine (RRL) and the Department of Family and Community Medicine (RLW, BS, RR), University of New Mexico, Albuquerque; the Department of Family and Community Medicine, University of California, San Francisco (MP); the Department of Family Medicine, University of Colorado, Denver (DF, WP, BP); Medicine @ Downtown, Albuquerque, New Mexico (MU); National Center for Primary Care, Morehouse School of Medicine, Atlanta, Georgia (ED); the Department of General Internal Medicine, The University of Texas MD Anderson Cancer Center, Houston, Texas (RJV); and the Department of Family and Community Medicine, Baylor College of Medicine, Houston, Texas (AEB). in a routine practice setting fail to achieve adequate pain relief. ${ }^{1,3}$ Chronic non-cancer pain (CNCP) causes significant morbidity, interfering with a patient's ability to perform activities of daily living, family life, and employment, and is associated with significant psychological stress. ${ }^{4-10}$ It is defined by

Funding: This project was funded in whole or in part with Federal funds from the National Institutes of Health, under Contract No. HHSN268200425211C, "ReEngineering the Clinical Research Enterprise," and by Grant No. D54HP00032-07-00 from the Health Resources and Services Administration.

Conflict of interest: none.

Corresponding author: Robert L. Williams, MD, MPH, Department of Family and Community Medicine, MSC09 5040; 1 University of New Mexico, Albuquerque, NM 87131 (E-mail: rlwilliams@salud.unm.edu). 
the American Society of the Interventional Pain Physicians as:

- Pain that persists beyond the usual course of an acute disease or a reasonable time for any injury to heal that is associated with chronic pathologic processes that cause continuous pain or pain at intervals for months or years

- Persistent pain that is not amenable to routine pain control methods ${ }^{11}$

Several studies have shown that there is substantial variability in the way clinicians approach and treat chronic pain. ${ }^{12,13}$ Multiple surveys have described the views and management habits of primary care clinicians in the treatment of $\mathrm{CNCP}$, though none has focused on clinicians who provide care to largely medically underserved patients. ${ }^{14-25}$ Primary care physicians are likely to report the management of CNCP as burdensome, ${ }^{14,16,24}$ and they frequently report concerns over abuse, diversion of prescribed opioids, addiction, inadequate training, and regulatory scrutiny, all which appear to create significant barriers to optimum treatment of chronic pain. ${ }^{14-19,21,23,24}$ Side effects of opioids and psychological factors in patients, such as poor coping, anxiety, and depression, have also been identified as barriers to effective treatment. ${ }^{14,15,18,21}$ One study reported that $35 \%$ of physicians were never willing to prescribe long-acting opioids for $\mathrm{CNCP} .{ }^{24}$ The lack of access to trained specialists in Pain or Addiction Medicine may also be a barrier to management of patients with chronic pain. ${ }^{20}$

The goal of this study was to gain a greater understanding about primary care clinicians' perspectives of the factors that affect clinicians' decisions related to CNCP management. The primary aims were to (1) describe views about CNCP among a sample of primary care clinicians caring mainly for medically underserved populations; (2) characterize these clinicians' reported $\mathrm{CNCP}$ management practices; and (3) describe primary care clinician perceptions of the context of care-those relationships, circumstances, resources, and surroundings that affect the care of patients with CNCP.

\section{Methods}

\section{Study Design}

A cross-sectional survey design was used to investigate the context of care for chronic pain in pri- mary care settings. The survey was conducted among primary care clinicians mostly practicing in low-income, medically underserved communities.

\section{Sample}

Clinicians from six primary care practice-based research networks were surveyed. Five of these networks are part of the PRImary care MultiEthnic Network (PRIME Net), a consortium of eight practicebased research networks: Research Involving Outpatient Settings Network: RIOS Net (New Mexico); the Colorado Research Network: CaReNet (Colorado); the Southeast Regional Clinicians' Network: SERCN (11 Southeastern states); the Southern Primary Care Urban Research Network: SPUR-Net (Houston); the San Francisco Bay Area Collaborative Research Network: SF Bay CRN (northern California); the Southwestern Ohio Ambulatory Research Network: SOAR-Net (southwestern Ohio); MetroNet (Detroit); and LA Net (Los Angeles) (http://hsc.unm.edu/som/primenet/). Clinicians in RIOS Net, CaReNet, SERCN, SPUR Net, and SF Bay CRN participated in this study. In addition, primary care clinicians from a sixth research network-BIGHORN (Colorado)—were surveyed. The majority of clinicians in these six networks practice in community health centers, Indian Health Service clinics, or academic practices serving low-income/underserved communities. Recruited clinicians are located in urban, suburban, and rural settings and the patient populations seen in these practices present with patterns of diagnoses typical of primary care. ${ }^{26}$ Each of the six network institutional review boards approved this study.

\section{Survey Instrument}

The questionnaire focused on contextual factors that might influence clinician approaches in the management of CNCP and included both closed- and openended items. Most closed-ended questions had categorical responses (e.g., strongly agree/agree/unsure/ disagree/strongly disagree; never/rarely/sometimes/ frequently). The survey was developed through a review of the existing literature, consultation with the lead author of a prior CNCP clinician survey, and iterative discussions with PRIME Net clinicians. ${ }^{24}$ We piloted the original long version, containing 26 questions and branching subquestions, among University of New Mexico Family Medicine and Internal Medicine faculty and residents, resulting in minor revisions. Average completion time was 13 minutes, 
based on timestamps for survey log-in. A second, shorter version of the questionnaire with four fewer main questions and more reduced subquestions was developed to enhance response rates among initial nonresponders. The remaining 22 questions were identical in both versions. Average completion time for this short form was 5 minutes. PDF copies of both versions are available on-line; readers are encouraged to refer to these for more detail about specific questions. ${ }^{27}$

\section{Data Collection}

E-mail announcements from leaders of each of the six research networks preceded an e-mail solicitation to network members directing them to a web-based long form version of the survey. This was followed by five e-mail solicitations with links to the long form questionnaire sent to nonresponders at weekly intervals. We mailed paper copies of the long form version to nonresponders. All solicitations offered drawings for gift certificates as response incentives. After these five e-mails and one postal solicitations, to enhance response rates, the shorter version of the on-line questionnaire was then made available and a series of four weekly e-mail solicitations were sent to remaining nonresponders.

The web-based versions of the survey were administered through the use of Opinio, a commercial product for designing and administering secure on-line surveys. ${ }^{28}$ Respondent identifiers (used for tracking nonresponders) were separated from the survey data. This design, while preserving respondent anonymity, eliminated the possibility of characterizing nonrespondents. We exported the anonymous data for statistical and qualitative analysis. Paper-based responses were doubly entered by RIOS Net staff into the database.

\section{Data Analysis}

Analysis of all quantitative data were done using SAS (version 9.1.3. SAS, Inc., Cary, NC). Categorical variables were collapsed (e.g., strongly agree/agree versus disagree/strongly disagree) for some analyses. $\chi^{2}$ tests were used to test for differences in (1) short-form/ long-form respondents; (2) selected outcomes of clinicians who had/had not participated in recent chronic pain continuing education; (3) selected outcomes of use of recommended office management systems (see below). The latter two comparisons were done to test whether these guideline recommended actions were associated with selected improvements.
We considered results statistically significant if the $P$ value was $<.05$. Narrative comments provided by clinicians to open-ended questions were analyzed by two investigators (RL, RW) who each independently reviewed the data, identifying common themes. The analysts then met to compare and resolve difference in the themes.

An "Office Score" was created to assess relationships of office systems for CNCP management to clinicians' perceptions of opioid efficacy and to perceived CNCP management burden. Clinicians were assigned one point for use of each of five recommended office systems, for a maximum of five points: pain assessment tool, controlled substance agreement (narcotic contract), refill policy, prescription log, and urine drug screening. Each clinician's Office Score was then compared with their responses to the questions "How many CNCP patients in your practice have benefited from long-term treatment with opioids in regard to significant improvement in functional outcome?" and "How much of a burden is the management of CNCP to you?"

\section{Results}

\section{Clinician Demographics}

The clinicians and their practice settings are described in Table 1. A total of 822 clinicians from 6 PBRNs (BIGHORN 25; CaReNet 174; RIOS Net 167; SERCN 68; SF Bay CRN 185; SPUR-Net 203) were invited to participate in the survey. Thirty invited clinicians were later determined to be ineligible (e.g., pediatrician seeing no patients with $\mathrm{CNCP}$ ) and were dropped from the sample. Of the remaining 792 clinicians, 497 total completed the questionnaire (response rate 63\%). Most of the respondents were from Colorado, New Mexico, Northern California, and Texas. Roughly onethird of the practices were located in inner cities, roughly one quarter in cities/suburbs and one quarter in medium $(<100,000$ population) or small ( $\leq$ 25,000 population) towns. Most of the respondents were physicians and most had more than 10 years' clinical experience. Of the clinicians, $78.8 \%(372)$ reported that they practiced family medicine and $20.8 \%$ (98) reported that they practiced general internal medicine. Four hundred twenty-seven $(86 \%)$ of the respondents used the long form, whereas 70 (14\%) respondents used the short form. Respondents to the short version of the survey were more likely to be affiliated with SPUR-Net, compared with those responding 
Table 1. Characteristics of Clinicians Participating in the Survey $(\mathrm{N}=497)$, Including Comparison of Those Responding to Either the Long-Version Survey or the Short Version*

\begin{tabular}{|c|c|c|c|c|}
\hline & \multicolumn{3}{|c|}{ Participants } & \multirow[b]{3}{*}{$P$ Value } \\
\hline & \multirow[b]{2}{*}{ Total } & \multicolumn{2}{|c|}{ Form } & \\
\hline & & Long & Short & \\
\hline \multicolumn{5}{|l|}{ Network } \\
\hline SF Bay CRN & $134(27.0 \%)$ & $118(27.6 \%)$ & $16(22.9 \%)$ & $<.001$ \\
\hline SERCN & $24(4.8 \%)$ & $17(4.0 \%)$ & $7(10.0 \%)$ & \\
\hline BIGHORN & $42(8.5 \%)$ & $38(8.9 \%)$ & $4(5.7 \%)$ & \\
\hline CaReNet & $93(18.7 \%)$ & $87(20.4 \%)$ & $6(8.6 \%)$ & \\
\hline RIOS Net & $125(25.2 \%)$ & $117(27.4 \%)$ & $8(11.4 \%)$ & \\
\hline SPUR-Net & $79(15.9 \%)$ & $50(11.7 \%)$ & $29(41.4 \%)$ & \\
\hline \multicolumn{5}{|l|}{ Geographic setting } \\
\hline Small town,$\leq 25 \mathrm{~K}$ pop. & $79(16.8 \%)$ & $74(18.4 \%)$ & $5(7.2 \%)$ & .05 \\
\hline Medium town, $>25 \mathrm{~K}$ pop. & $40(8.5 \%)$ & $36(9.0 \%)$ & $4(5.8 \%)$ & \\
\hline City $100 \mathrm{~K}-500 \mathrm{~K}$ pop. & $54(11.5 \%)$ & $45(11.2 \%)$ & $9(13.0 \%)$ & \\
\hline Urban/Suburban $>500 \mathrm{~K}$ pop. & $134(28.5 \%)$ & $116(28.9 \%)$ & $18(26.1 \%)$ & \\
\hline Urban inner city $>500 \mathrm{~K}$ pop. & $164(34.8 \%)$ & $131(32.6 \%)$ & $33(47.8 \%)$ & \\
\hline \multicolumn{5}{|l|}{ Clinic type } \\
\hline Community health center & $200(42.2 \%)$ & $169(41.7 \%)$ & $31(44.9 \%)$ & .03 \\
\hline Indian health service & $34(7.2 \%)$ & $34(8.4 \%)$ & $0(0.0 \%)$ & \\
\hline Solo practitioner & $23(4.9 \%)$ & $21(5.2 \%)$ & $2(2.9 \%)$ & \\
\hline University practice & $125(26.4 \%)$ & $101(24.9 \%)$ & $24(34.8 \%)$ & \\
\hline Other group practice & $92(19.4 \%)$ & $80(19.8 \%)$ & $12(17.4 \%)$ & \\
\hline \multicolumn{5}{|l|}{ Clinician training } \\
\hline MD & $402(85.0 \%)$ & $339(83.9 \%)$ & $63(91.3 \%)$ & .75 \\
\hline DO & $14(3.0 \%)$ & $13(3.2 \%)$ & $1(1.4 \%)$ & \\
\hline NP & $24(5.1 \%)$ & $22(5.4 \%)$ & $2(2.9 \%)$ & \\
\hline $\mathrm{PA}$ & $31(6.6 \%)$ & $28(6.9 \%)$ & $3(4.3 \%)$ & \\
\hline Other degree & $2(0.4 \%)$ & $2(0.5 \%)$ & $0(0.0 \%)$ & \\
\hline \multicolumn{5}{|l|}{ Years since completed training } \\
\hline N/A & $29(6.2 \%)$ & $25(6.2 \%)$ & $4(5.8 \%)$ & .85 \\
\hline$<5$ years & $77(16.3 \%)$ & $65(16.2 \%)$ & $12(17.4 \%)$ & \\
\hline 5 to 10 years & $101(21.4 \%)$ & $83(20.6 \%)$ & $18(26.1 \%)$ & \\
\hline 11 to 20 years & $145(30.8 \%)$ & $125(31.1 \%)$ & $20(29.0 \%)$ & \\
\hline$>20$ years & $119(25.3 \%)$ & $104(25.9 \%)$ & $15(21.7 \%)$ & \\
\hline
\end{tabular}

*Totals for some demographic characteristics do not equal 497 because of missing data.

SF Bay CRN, San Francisco Bay Area Collaborative Research Network; SERCN, Southeast Regional Clinicians' Network; BIGHORN, Health Outcomes Research Network; CaReNet Colorado Research Network; RIOS Net, Research Involving Outpatient Setting Network; SPUR-Net, Southern Primary Care Urban Research Network; MD, doctor of medicine; DO, doctor of osteopathy; NP, nurse practioner; PA, physician assistant.

to the long form, and consistent with SPUR-Net membership they were more likely to be practicing in innercity, university-affiliated practices. Short-version respondents were significantly less likely to ever prescribe opioids ( $29 \%$ vs. $13 \%, P=.003$ ), and therefore were less likely to use controlled substances agreements or to report having had patients divert opioids. In all other ways, their responses were neither statistically nor clinically different from long form respondents.

\section{Major Data Themes}

1. Many Barriers Exist to the Treatment of CNCP, Including Many Uncertainties in Optimal Management Forty-seven percent of clinicians in this sample reported mental health services were not financially available to their patients, and $34 \%$ reported substance abuse counselors were not available. Other consultants, such as pain management specialists, were equally unavailable (Table 2). 
1. Many barriers exist to the treatment of CNCP, including many uncertainties in optimal management

Behavioral health care not financially accessible to CNCP patients* (\% yes)

Pain specialist not financially accessible to CNCP patients* (\% yes)

Complementary/alternative medicine providers (e.g., acupuncture, herbalists) not financially accessible to CNCP patients* (\% yes)

Need for more CNCP continuing medical education (\% agree/strongly agree)

Long-acting opioids are over-utilized for many CNCP patients in my community (\% agree/strongly agree)

Long-acting opioids are under-utilized for many CNCP patients in my community (\% agree/strongly agree)

2. For many patients, CNCP is a complex biopsychosocial condition for which a multidisciplinary approach is needed

Experience discomfort in managing CNCP because of: (\% some/moderate/ much discomfort)

Patient behavioral health issues*

Lack of a defined mechanism of pain*

Lack of full work-up for cause of pain*

Observe common reasons for uncontrolled pain in CNCP patients: (\% agree/ strongly agree)

Depression, anxiety, other mental health disorders

Alcohol or other substance use disorders

Poor coping skills for pain

Poor coping skills for other life stressors*

Use massage therapy (\% ever used)

Use acupuncture (\% ever used)

Find cognitive behavioral therapy helpful (\% somewhat/very helpful)

Find biofeedback, meditation, or relaxation therapy helpful (\% somewhat/very helpful)

3. Prescription opioid abuse is a serious problem

Report patients ever divert opioids (\% yes)

Report having had patients with severe adverse event due to CNCP opioid use (unintentional life-threatening event or death, or suicide) (\% yes)

Prescribing opioids limited by (\% sometimes/frequently)

Concern over opioid side effects*

Concern over development of dependence*

Concern over development of addiction*

Concern over malingering/secondary gain*

Prescribe long-acting opioids

No

Don't initiate but continue Rx prescribed by others

Initiate and continue

4. Significant effort is required to properly assess for efficacy, side effects, and aberrant drug related behavior related to chronic opioid treatment

Report burden to self in managing CNCP (\% fair/large/biggest burden)

Report burden to practice in managing CNCP (\% fair/large/biggest burden)

Report being "troubled" prescribing long-acting opioids for: (\% yes)

Chronic neck/back pain without defined mechanism*

Chronic headaches*

Chronic pelvic pain*

Chronic abdominal pain*
426

421

422

$199(47 \%)$

$143(34 \%)$

$172(41 \%)$

$190(45 \%)$

$204(44 \%)$

$212(43 \%)$

$152(31 \%)$

489

$383(96 \%)$

$371(91 \%)$

$377(93 \%)$

$439(94 \%)$

$354(76 \%)$

$402(86 \%)$

$371(93 \%)$

$379(78 \%)$

$354(73 \%)$

$330(67 \%)$

$306(62 \%)$

$341(70 \%)$

$161(33 \%)$

$329(80 \%)$

$292(70 \%)$

$356(86 \%)$

$320(77 \%)$

75 (15\%)

$80(17 \%)$

$329(68 \%)$

497

$400(80 \%)$

$436(88 \%)$

400

$236(59 \%)$

$238(60 \%)$

$201(50 \%)$

221 (55\%)

Continued 


\begin{tabular}{|c|c|c|}
\hline & $\begin{array}{l}\text { Total Number of } \\
\text { Respondents }\end{array}$ & $\begin{array}{l}\text { Total Positive } \\
\text { Responses, } \mathrm{n}(\%)\end{array}$ \\
\hline \multicolumn{3}{|l|}{ Use practice systems for CNCP management: (\% yes) } \\
\hline Use controlled substance agreements & 472 & $407(86 \%)$ \\
\hline Use urine drug screens & 470 & $307(65 \%)$ \\
\hline Use CNCP monitoring tool & 474 & $104(22 \%)$ \\
\hline \multicolumn{3}{|l|}{$\begin{array}{l}\text { 5. Perceived benefits of opioids to some patients and commitment to their } \\
\text { patients influences clinicians to continue }\end{array}$} \\
\hline View CNCP as important clinical problem (\% agree/strongly agree) & 495 & $471(95 \%)$ \\
\hline $\begin{array}{l}\text { Concerned about poor quality of life for CNCP patients due to pain* } \\
(\% \text { agree/strongly agree })\end{array}$ & 414 & $401(97 \%)$ \\
\hline $\begin{array}{l}\text { Concerned that many } \mathrm{CNCP} \text { patients receive inadequate pain control* } \\
(\% \text { agree/strongly agree })\end{array}$ & 412 & $266(65 \%)$ \\
\hline $\begin{array}{l}\text { Concerned about uncontrolled pain due to lack of access to resources } \\
\text { (\% agree/strongly agree) }\end{array}$ & 469 & $343(73 \%)$ \\
\hline $\begin{array}{l}\text { Report benefit from patient education (e.g., handouts, web links) } \\
\text { (\% yes) }\end{array}$ & 493 & $359(73 \%)$ \\
\hline Report some CNCP patients benefit from opioid treatment* (\% yes) & 405 & $316(78 \%)$ \\
\hline
\end{tabular}

${ }^{*}$ Question included on long-form version of survey only.

$†$ Number of respondents does not match total sample because of missing data.

CNPC, chronic non-cancer pain.

“. . . many who really need psychiatric care/counseling but do not have access end up on opioids for the complicated interface of physical pain and unremitting psychosocial stressors/emotional pain."

Clinicians expressed concern about the limited evidence to guide safe and effective prescribing of opioids.

"[What influences me not to prescribe opioids is the] lack of randomized controlled trials showing benefit of opioids for CNCP."

The uncertainties about optimal pain management were reflected in the fact that $44 \%$ of participants agreed with the statement that "long-acting opioids are over-utilized in my community," whereas $31 \%$ agree that "long-acting opioids are under-utilized in my community." Similarly, 41\% felt a colleague in their practice prescribes too many opioids for CNCP, and 33\% felt that a colleague in their practice prescribes too few opioids.

"[My discomfort is from the] inconsistent use of narcotics within our large group practice with no set way of managing process of care for patients with CNCP.”

\section{For Many Patients, CNCP Is a Complex Biopsychosocial Condition for Which a Multidisciplinary Approach Is Needed} Clinicians in this sample expressed discomfort about many aspects of CNCP management. Contributing factors chosen most often were behavioral/mental health issues (96\% clinicians reported this as a factor), lack of full work-up for the cause of the chronic pain (93\%), lack of defined mechanism for the pain (91\%), lack of demonstrated pain behaviors (85\%), lack of available consultants to assist with management (77\%), lack of support services (72\%), and their own lack of training (64\%).

Underlying behavioral health issues was selected as the most important source of discomfort for $43 \%$ of clinicians when managing patients with CNCP.

"I do not find treating these patients very fulfilling. They tend to come with behavioral, mental health issues that suck time and energy away from other patients."

"A high percentage of patients who have severe lifelong trauma are actually unable to distinguish physical and emotional pain-I am quite hesitant about starting down the road of opiates."

"I think opioids are often used by patients for overall life dissatisfaction, ennui, boredom and depression."

Understanding this complex biopsychosocial nature of CNCP for many patients, clinicians readily used or referred for a variety of treatment modalities for their patients. Massage therapy was used by $78 \%$ of clinicians, acupuncture by $73 \%$, and some other type of alternative treatment by $65 \%$ of clinicians (for example, yoga, meditation, biofeedback, trigger point, chiropractic or osteopathic manipulation). Clinicians who reported participation 
in CNCP continuing medical education (CME) within the last 5 years also reported higher use of cognitive behavioral therapy $(41 \%$ vs. $29 \%, P=$ $.04)$, and biofeedback/meditation/relaxation training $(38 \%$ vs. $21 \%, P<.001)$.

\section{Prescription Opioid Abuse Is a Serious Problem}

Concern over addiction and diversion were the most frequently cited factors by these clinicians for influencing them not to prescribe opioids. Moreover, $70 \%$ of respondents reported having had a patient probably or definitely divert opioids (Table 2). Thirty-three percent of clinicians reported having a patient with a probable or definite life-threatening event, death, or suicide related to an opioid they had prescribed.

"I [prescribed narcotics] more in past. Ran into variety of problems [including]diversion. . . I am moving out of the business of working with these patients as much as I possibly can."

"One of the challenges of treating CNCP that I have come across is that I get to be known to the whole community as a physician who prescribes narcotics. This attracts a larger number of difficult and challenging patients with comorbid psychiatric disorders and prescription medication abusers."

And, perhaps most simply put: "My area of greatest concern is concurrent substance abuse."

\section{Significant Effort Is Required to Properly Assess for Efficacy, Side Effects, and Aberrant Drug-Related Behavior Related to Chronic Opioid Treatment}

Eighty-one percent of the clinicians in this sample reported the management of $\mathrm{CNCP}$ as a burden to them, and $88 \%$ reported it as a burden to their practice. Fifteen percent of the respondents did not prescribe long-acting opioids under any circumstance and another $17 \%$ will not initiate opioids (but will continue them when prescribed by another clinician). Controlled substance agreements ("opioid contracts") were used by $86 \%$ of clinicians (sometimes/always), and urine drug screens were used by $65 \%$ (sometimes/always). Only $22 \%$ of clinicians, however, regularly used a CNCP monitoring tool (pain assessment tool, controlled substance agreement/narcotic contract, refill policy, prescription log, urine drug screening). The time required for $\mathrm{CNCP}$ management was mentioned by some clinicians.
"[My discomfort comes from the] lack of time for a full evaluation and lack of timely referral services for full evaluation of pain. .."

For those clinicians who managed to incorporate chronic pain monitoring and assessment tools into their practice, they did perceive improved efficacy of care, yet at the cost of some increase in provider/practice burden. This is especially true for clinicians who prescribed opioids and is illustrated in Figure 1. A graded effect is noted when the Office Systems Score is compared with the perception of opioid efficacy $(P<.0001)$ as well as to practice burden $(P=.03)$.

Better access to support services and consultants was associated with a reduced sense of burden for these clinicians, as was limiting the prescribing of opioids. Clinicians with access to a pain clinic were significantly less likely to report $\mathrm{CNCP}$ as a large burden ( $6 \%$ vs. $27 \%, P<.001)$.

Clinicians who reported participation in CNCP CME within the last 5 years $(73 \%$ of the participants) reported higher use of a pain assessment tool (27\% vs. $12 \%, P<.001)$, narcotic contract $(88 \%$ vs. $78 \%, P=.02$ ), and refill policy ( 92 vs. $85 \%, P=$ $.02)$. They also reported a higher degree of functional improvement with opioids ( 82 vs. $65 \%, P=$ $.005)$ and more familiarity with state guidelines (50 vs. $22 \%, P<.0001)$. However, participation in CNCP CME in the last 5 years had no effect on perceived burden.

\section{Perceived Benefits of Opioids to Some Patients and Commitment to Their Patients Influence Clinicians to Continue}

Not all aspects of CNCP care were negative. The majority of clinicians in the sample recognized the importance of $\mathrm{CNCP}$ care in primary care practice, and some could achieve a sense of success and fulfillment in working with these patients.

Ninety-five percent of clinicians reported CNCP is important relative to the other problems they see. Seventy-eight percent of respondents believed that at least some of their patients have benefited from chronic opioid treatment. In response to the question "What factors influence you to prescribe opioids," one clinician responded:

"[It is the] long-standing relationships with many of my patients, level of trust, and [their] desire to improve function."

Likewise, other clinicians express satisfying experiences with $\mathrm{CNCP}$ care: 
Figure 1. Effects of Office Score on perceived opioid efficacy and burden for opioid prescribing clinicians. Clinicians were assigned one point for use of each of five office systems: Pain Assessment Tool, Controlled Substance Agreement (Narcotic Contract), Refill Policy, Prescription log, and Urine Drug Screening.

\section{Effect of Office Score on Perception of Opioid Efficacy}
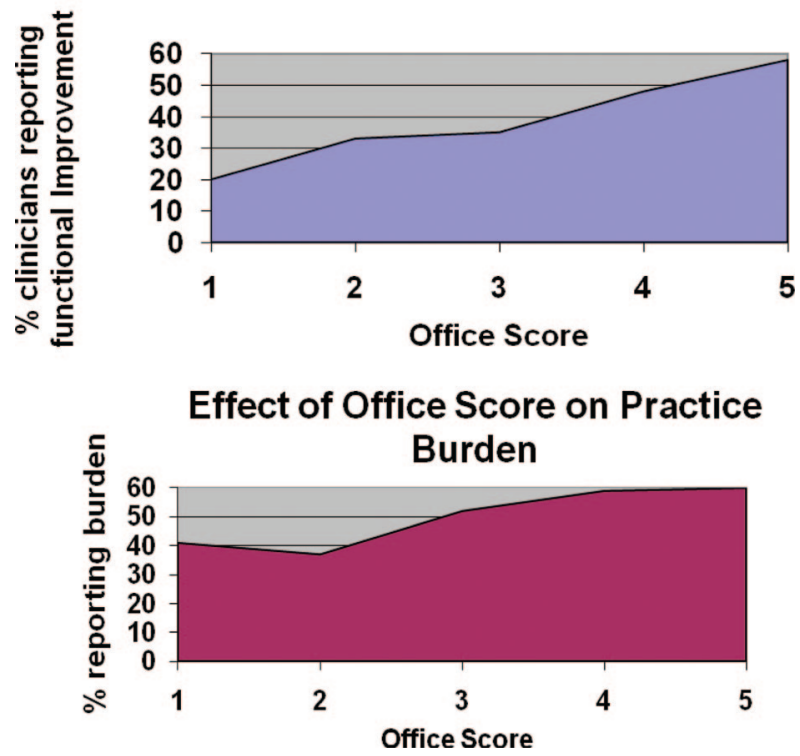

"The population I serve has, in general, little problem with drug seeking behavior and so many patients are stoic."

"There is a group of CNCP patients that I treat/ manage in association with others with good results/ improvement in functioning and overall feel quite good with management plans."

Despite the burdens described above, only $31 \%$ of the participants agreed with the statement, "my practice is not set up to accommodate patients needing the regular follow-up care required by CNCP patients," and 64\% did not agree with the statement that "there are too many competing demands for me to be able to attend to the regular follow-up care required by CNCP patients.”

\section{Discussion}

Chronic non-cancer pain is a complex and challenging problem affecting multiple domains of well-being and quality of life. When patients with CNCP seek care, clinicians should carefully weigh the anatomic, pathophysiologic, behavioral, social, and spiritual contributions to their suffering. In the current construct of most primary care practices, this is not an easy goal to accomplish. These challenges were compounded for the clinicians in this study, who primarily cared for medically underserved communities, due to a lack of behavioral and consultative resources to assist with care.

In their survey responses and accompanying narrative comments, the clinicians in this sample clearly emphasized the challenges that patients with chronic non-cancer pain present. An alarming 33\% of these clinicians report having had a patient who had a severe outcome from opioids they had prescribed (suicide or other death, or life-threatening event). Some $70 \%$ reported likely diversion of prescribed opioids. The often difficult nature of interactions with patients with CNCP, lack of precision in the diagnosis of causes of their patients' chronic pain, the lack of evidence basis for management recommendations, and limited availability of pain management services all likely contributed to the finding that more than $80 \%$ of the clinicians reported care of CNCP patients is a burden to them and/or their practices. The prominent role of comorbid behavioral health issues in many CNCP patients, coupled with the reported lack of resources to assist with behavioral health also increases burden. Yet, despite this, many clinicians reported experiencing satisfaction with caring for CNCP patients.

This study underlines the difficulty in treating the subjective nature of chronic pain, often in the absence of clear objective diagnostic findings. This leads to further divergence in practice patterns when each individual physician must evaluate a patient's subjective pain and arrive at a treatment plan. Clinical decisions are based on interpretation of subjective symptoms, prior experiences, and judgment on the risk/benefits of initiation/continuation of opioid treatment. This leads to very different decisions among physicians for the same clinical presentation.

\section{Guidelines and Previous Research}

The results of this survey are generally concordant with major themes and principles described in the American Pain Society and American Academy of Pain Medicine Opioid Treatment Guideline and highlight important barriers to effective treatment of CNCP in primary care. ${ }^{29}$ The guidelines stress the importance of careful assessment and monitoring of patients on chronic opioid therapy, the difficulties of aberrant drug-related behavior, the 
value of consultation with mental health or addiction specialists, the potential value of psychotherapeutic intervention, and the uncertainty associated with patient selection and value of chronic opioid therapy. As the authors of these guidelines note, unfortunately little evidence exists to assist clinicians in this endeavor-they rated only 4 of their 25 recommendations as supported by even moderate quality evidence. ${ }^{29}$ Furthermore, effective application of the guidelines would require a level of resources that these clinicians report are unavailable.

Provider education and training may have an important role in encouraging the adoption of "best practices" for the management of $\mathrm{CNCP}$, as recent participation in CNCP CME was associated with higher use of guideline-recommended office systems to manage CNCP. However, while use of an increasing number of office management tools for $\mathrm{CNCP}$ care was associated with increased perceived efficacy of opioids in $\mathrm{CNCP}$ among these clinicians, their reported effects on burden leaves open the question of their feasibility.

The findings of this study are also consistent with those of earlier surveys reporting clinicians' views about the burden of management of CNCP, concerns about side effects of chronic opioid therapy, issues of psychiatric co-morbidities in CNCP patients, and limited access to consultation services. ${ }^{14,16,21,23-25}$ In contrast to earlier surveys, this study provides a more indepth perspective of these views, including the frequent occurrence of life-ending or life threatening consequences of chronic opioid therapy, and the lack of mental health, addiction, or pain consultation. Despite this, the persistence of most clinicians in attempting to help their patients with CNCP came through clearly in this study. On the other hand, the substantial percentage of clinicians who reported not prescribing opioids-as has been previously noted in one study ${ }^{24}$ - may represent a workforce casualty of the burden of CNCP management.

Although regulatory oversight emerged in other surveys as an important factor driving clinician CNCP decision making, it was not seen as a common concern among clinicians in this study. This may reflect the fact that this study was conducted in a context of widespread concern about inadequate treatment of pain. More recent publicity and proposed regulation related to overprescribing of opioids with resulting fatalities could reinsert this issue into clinicians' CNCP decision making. ${ }^{30}$

\section{Direction Forward}

The results of this study support those of a small study of family physicians using a Delphi approach to identify 4 approaches to improved care of CNCP: (1) practice guidelines; (2) changes in opioid refill processes; (3) self-management support and access to alternative treatments; and (4) nurse case management. ${ }^{31}$ These results as well as those from the current study both suggest applicability of the Chronic Care $\mathrm{Model}^{32}$ to management of CNCP. The Chronic Care Model, with its emphasis on 6 components of care-self-management support, clinical information systems, delivery system redesign, decision support, health care organization, and community resources-appears well suited for $\mathrm{CNCP}$, and may be the best answer to improved CNCP care, should resources be aligned to make the necessary system of care changes.

\section{Limitations}

The findings of this study are limited by the fact that the PRIME Net consortium within which it was conducted is composed largely of clinicians in medically underserved communities. This may have resulted in greater emphasis on the lack of available relevant consultation. However, similar concerns have been seen in previous surveys on this topic. Furthermore, the wide geographic coverage of the PRIME Net consortium suggests this problem is widespread. The study design did not include validation of clinicians' reported experiences. For example, clinicians taking the extra effort to follow guidelines or use care management systems may have been inclined to report greater efficacy of opioids as a result of "wishful thinking." It is important to recall as well that associations, such as those reported here between office systems and perceived efficacy of opioids, do not prove causation. Finally, the cross-sectional survey design may have resulted in biased responses, for example by greater participation in the survey by clinicians frustrated at the time with $\mathrm{CNCP}$ care. 


\section{Conclusions}

This study has provided further understanding of CNCP management in primary care, depicting the frustration, unease, lack of evidence, lack of resources, and bad outcomes, yet, persistence in attempting to provide care. The challenge now is to explore how new models of care for CNCP can enhance its management both for patients and clinicians. Can the Chronic Care Model and its close relative, the Patient-Centered Medical Home, make resources available to provide the comprehensive care and the systematic approach that many CNCP patients need to address their pain? The nature of chronic pain and the anguish and urgency it compels serve as a siren for the need for better chronic care infrastructure in primary care.

We acknowledge and appreciate the insights that each of the participating PRIME Net clinicians provided us. We appreciate the important contributions to this study provided by Anthony Adams and Laurie McPherson, who assisted with survey data collection.

\section{References}

1. Whitten CE, Evans CM, Cristobal K. Pain management doesn't have to be a pain: working and communicating effectively with patients who have chronic pain. Perm J 2005;9:41-8.

2. Turk DC. Treatment of chronic pain: clinical outcomes, cost effectiveness and cost benefits. Drug Benefit Trends 2001;13:36-8.

3. Cherney NI, Portenoy RK. The management of cancer pain. CA Cancer J Clin 1994;44:262-303.

4. Krein SL, Heisler M, Piette JD, et al. Overcoming the influence of chronic pain on older patients' difficulty with recommended self-management activities. Gerontologist 2007;47:61-8.

5. Gureje O, Von Korff M, Simon GE, Gater R. Persistent pain and well-being: a World Health Organization study in primary care. JAMA 1998;280:147-51.

6. Elliott AM, Smith BH, Penny KI, et al. The epidemiology of chronic pain in the community. Lancet 1999;354:1248-52.

7. Brooks PM. The burden of musculoskeletal disease: a global perspective. Clin Rheumatol 2006;25:778-81.

8. Smith BH, Elliott AM, Chambers WA, et al. The impact of chronic pain in the community. Fam Pract 2001;18:292-9.

9. Crosby FE, Colestro J, Ventura MR, Graham K. Survey of pain among veterans in Western New York. Pain Manag Nurs 2006;7:12-22.

10. Duong BD, Kerns RD, Towle V, Reid MC. Identifying the activities affected by chronic nonmalignant pain in older veterans receiving primary care. J Am Geriatr Soc 2005;53:687-94.
11. Trescot AM, Helm S, Hansen H, et al. Opioids in the management of chronic non-cancer pain: an update of American Society of Interventional Pain Physicians' (ASIPP) guidelines. Pain Physician 2008;11: S5-S62.

12. Green CR, Wheeler JR, LaPorte F, et al. How well is chronic pain managed? Who does it well? Pain Med 2002;3:56-65.

13. Ballantyne JC, Mao, J. Opioid therapy for chronic pain. N Engl J Med 2003;349:1943-53.

14. Upshur CC, Luckmann RS, Savageau JA. Primary care provider concerns about management of chronic pain in community clinic populations. J Gen Intern Med 2006;21:652-5.

15. Stannard C, Johnson M. Chronic pain management: can we do better? An interview-based survey in primary care. Curr Med Res Opin 2003;19:703-6.

16. Dobscha SK, Corson K, Flores JA, et al. Veterans Affairs primary care clinicians' attitudes toward chronic pain and correlates of opioid prescribing rates. Pain Med 2008;9:564-71.

17. Boulanger A, Clark AJ, Squire P, et al. Chronic pain in Canada: have we improved our management of chronic noncancer pain? Pain Res Manag 2007;12:39-47.

18. Morley-Forster PK, Clark AJ, Speechley M, Moulin DE. Attitudes toward opioid use for chronic pain: a Canadian physician survey. Pain Res Manag 2003;8: 189-94.

19. Nwokeji ED, Rascati KL, Brown CM, Eisenberg A. Influences of attitudes on family physicians' willingness to prescribe long-acting opioid analgesics for patients with chronic nonmalignant pain. Clin Ther 2007;29:2589-2602.

20. Mitchinson AR, Kerr EA, Krein SL. Management of chronic noncancer pain by VA primary care providers: when is pain control a priority? Am J Manag Care 2008;14:77-84.

21. Bhamb B, Brown D, Hariharan J, et al. Survey of select practice behaviors by primary care physicians on the use of opioids for chronic pain. Curr Med Res Opin 2006;22:1859-65.

22. Jamison RN, Gintner L, Rogers JF, Fairchild DG. Disease management for chronic pain: barriers of program implementation with primary care physicians. Pain Med 2002;3:92-101.

23. Ponte CD, Johnson-Tribino J. Attitudes and knowledge about pain: an assessment of West Virginia family physicians. Fam Med 2005;37:477-80.

24. Potter M, Schafer S, Gonzalez-Mendez E, et al. Opioids for chronic nonmalignant pain: attitudes and practices of primary care physicians in the UCSF/Stanford Collaborative Research Network. J Fam Pract 2001;50:145-51.

25. O'RorkeJE, Chen I, Genao I, et al. Physicians' comfort in caring for patients with chronic nonmalignant pain. Am J Med Sci 2007;333:93-100.

26. Binns HJ, Lanier D, Pace WD, et al. Describing primary care encounters: the Primary Care Network 
Survey and the National Ambulatory Medical Care Survey. Ann Fam Med 2007;5:39-47.

27. PRIME Net Chronic non-malignant pain survey. Long form version available at: http://hsc.unm.edu/ rios/docs/Chronic_Non-Malignant_Pain_(CNMP)_ Survey.pdf. Short form version available at: http:// hsc.unm.edu/rios/docs/SHORT_Version_Chronic_ Non-Malignant_Pain_(CNMP)_Survey.pdf.

28. Object planet. Opinio software. Available at: http:// www.objectplanet.com/opinio/. Accessed December 28, 2010.

29. Chou, R, Fanciullo GJ, Fine PG, et al. Opioid treatment guidelines: clinical guidelines for the use of chronic opioid therapy in chronic noncancer pain. J Pain 2009; 10:113-30.

30. Meier B. Move to restrict pain killers puts onus on doctors. New York Times. July 28, 2010. Available at: http://www.nytimes.com/2010/07/29/business/29pain. html. Accessed on December 28, 2010.

31. Garufi Clark L, Upshur CC. Family Medicine physicians' views of how to improve chronic pain management. J Am Board Fam Med 2007;20:479-82.

32. Bodenheimer T, Wagner EH, Grumbach K. Improving primary care for patients with chronic illness. JAMA 2002;288:1775-9. 